\title{
Augenärztliche Gesellschaft in Hamburg
}

Sitzung vom 25. November 1927

Hamann: Bericht über einen Fall von Aneurysma der rechten Carotis

interna, dessen Symptome - Kopfschmerzen, Ohrensausen, Doppelbilder - sich erst im Herbst 1926 nach einem vor vier Jahren crlittenen Unfall bemerkbar machten. Objektiv bestanden Horizontal- und Vertikal-nystagmus, Doppelbilder im Sinne einer rechtsseitigen Abduzensparese und beiderseitige Stauungspapille von 3-4 Dptr. Prominenz. Nach Unterbindung der rechten Carotis interna Rückgang der Krankheits-erscheinungen - Aufhören des Doppeltsehens und allmählicher Ruck-

31()

Augenarztliche Gcsellschaft in Hamburg.

gang der Stauungspapille - und subjektives Wohlbefinden. Das Aneu-rysma muß den Symptomen nach in der Gegend des Austritts der Ca-rotis interna aus dem Felsenbein und im Bereich des Sinus cavernosus angenoinmen werden.

Emma Schindler stellt eine schmächtige 31 jährige Frau mit Myastheuia gravis pseudoparalytica vor. In der Jugend keine erhebliehen Krank-heiten, Wassermann negativ. Vor sieben Jahren trat das Leiden während des Stillens nach normaler Geburt zuerst auf und wiederholte sich seit dieser Zeit in verschiedenen Abständen (1, 1/2, 1/4 Jahr). Es äußert sich in tage- bzw. wochenlang anhaltenden Lähmungen der äußeren Augen-muskeln auf einem oder gleichzeitig auf beiden Augen. Zeitweise besteht beiderseitige totale Ptosis. Im Gegensatz dazu ist die innere Muskulatur des Auges funktionstüchtig. Seit zwei Jahren zunehmende Schwäche der oberen und unteren Extremitäten. Neurologisch: Lebhafte Reflexe, koordinierte langsame Bewegungen, mvasthenische Reaktion nach Jolly negativ. Keine Bulbärsyrnptome, die sonst bei dem Krankheitsbild meist vorhanden sind.

Fall von rezidiviorend $<>$ r Okulomotoriuslähmung.

$>$ 3Jähriger junger Mann ohne neuropathische Veranlagung erkrankte aus bestem Wohlbefinden heraus vor elf Monaten und erneut vor drei Monaten an heftigen rechtsseitigen, zwei resp. vier Tage anhaltenden Kopfschmerzen mit Erbrechen, die von kompletter Okulomotoriuslähmung (externa wie interna) abgelöst wurden. Das Außergewöhnliche ist beide Male die vier Wochen lange Dauer der Lähmung bis zum Beginn der langsam fortschreitenden Wiederherstellung, die nach sechs Wochen vollkommen war. Bei dem ersten Anfall wurden Schwitzen und Jod-kali therapeutisch angewandt, das zweite Mai erfolgte die Genesung ohne jede Therapie. Eingehende Untersuchungen nach alien Richtungen führten nicht zur Klärung der Atiologie.

Mylius berichtet nach kurzer Besprechung der Apparatur und ' $\Gamma$ echnik und nach kurzer Charakterisierung der Strahlen über experi-mentelle Yersuchsergebnisse über die Wirkung von Biu·kystrahlen Grenz· strahlen) auf (las Auge des Kaninchens. Vorausgeschickte Untersuchungen über die Beeinflussung des Bakterienwachstums auf Nährböden lassen bei den Tuberkelbazillen, den Bazillen der Typhus-, Coli- und Dys-enteriereihe, sowie bei Strepto-, Staphylo- und Pneumokokken keine Hemmung des Wachstums durch die Buckystrahlen erkennen, selbst nicht in Dosen von mehreren $100^{\circ} 0$ der HED. Am Auge ergab sich fol-gendes: 
1. Die Hornhaut des bestrahlten Auges ist für in den Bindehaut-sack gebrachtes Fluoreszin besser durchlässig als die des Kontrollauges. 2. Der Flüssigkeitswechsel des Auges, der durch eine Röntgenbestrahlung stets beeinträchtigt wird, was daraus zu schließen ist, daß subkutan injiziertes Fluoreszin früher im Kammerwasser des bestrahlten Auges als im Kontrollauge erscheint und daß der Eiweißgehalt des Kammer-wassers des bestrahlten Auges höher ist als im Kontrollauge, wird durch Buckybestrahlung nicht nachweisbar beeinträchtigt. 3. Experimentell gesetzte Epitheldefekte und infizierte Ulcera corneae heilen etwas schneller und mit etwas zarterer Narbe als auf dem nicht bestrahlten Auge. 4. Experimentell gesetzte Hornhauttuberkulose (mit humanem TbcAugenärztliche Gesellschaft in Hamburg.

\section{7}

Stamm) wird durch die Bestrahlung nicht sehr wesentlich beeinflußt. Es tritt wohl raschere Vaskularisierung und zunächst auch stärkere kollaterale Durchtränkung der Hornhaut nach der Bestrahlung mit an-schließender beschleunigter Vernarbung ein, doch ist der Endausgang (nach 4--9 Monaten) kein wesentlich anderer wie auf dem Kontrollauge und auf den Augen überhaupt nicht bestrahlter Kaninchen. Es muß allerdings erwähnt werden, daß die Infektion sehr mäßig war (Hornhaut-quaddel mit einer'Lösung von $1 \mathrm{mg}$ Tbc. in $1 \mathrm{ccm}$ Kochsalzlösung). Eine zufällig entstandene Bindehauttuberkulose heilte sehr schnell unter der Bestrahlung ab, doch fehlte die Kontrolle. Eine Iristuberkulose wurde nicht beeinflußt.

Normale Kaninchenaugen ließen selbst nach mehrmaliger Bestrahlung mit mehreren $100^{\circ} 0$ der HED. bei einer Beobachtungszeit von fünf Monaten keine pathologischen Veränderungen erkennen. Am Menschen wurden bisher erst zwei Ulcera serpentia und ein tuberkulöses Infiltrat bestrahlt, so daß noch kein Urteil abgegeben werden kann. Die Versuche sollen fortgesetzt werden, da die Buckybestrahlung der Röntgenbestrahlung wegen der geringeren Tiefenwirkung vorzuziehen sein würde, selbst wenn die Erfolge nicht besser als bei der Röntgenbestrahlung sein würden.

51. Versammlung des Vereins rheinisch-westfälischer Augenärzte am 27. November 1927 in

Düsseldorf.

Fortbildungsvortrag von Professor Dr. Krückmann (Berlin): tlber Wirkung der

Zirkulationszustände des Auges unter Berücksichtigung kli- nischer Betrachtung, mit stereoskopischer Betrachtung von Injektions-präparate $\pi$.

Sodann Vorträge:

1. von Szily: Zur Behandlung des Keratokonus.

Vortragender kombiniert die Kaustik der Keratokonusspitze mit der Goldchloridtätowierung nach Knapp. Er hat mit dieser Methode in einer Reihe von Fallen günstige optische Ergebnisse erzielt, die zahlen-mäßig belegt werden.

2. Poos: Zur Frage der Ergotaminwirkung am Auge.Vortragender studierte die Wirkungsweise des Ergotamins, auf

d. dessen Bedeutung für das Glaucom besonders Thiel hingewiesen hat auf das Kaninchenauge und fand

daß es hierbei als ein typisches Reizmittel des Sympathicus von adrenalinähnlichem Charakter auftritt. Das Menschen-auge scheint sich

soweit Verf. das nach seinen Versuchen bisher über-blicken kann

ähnlich zu verhalten. Die relativ schwache mydriatische Wirkung des Ergotamins am menschlichen Auge führt er auf eine gleich-zeitige Reizwirkung auf den Parasympathicus zurück. Bei drei Fallen von denen er gleichzeitig eine Akkommodationskurve aufnahm 
wurde die Kokainwirkung auf den Ziliarmuskel durch die Ergotaminwirkung unterdrückt und auf dieser Seite ein Ziliarmuskelkrampf wie nach Eserin für einige Stunden beobachtet iníolge einem starken Uberwiegen der

Zeitschrift für Augenhei1/8unde. Bd. 64. Heft 4/5. 21 